\title{
USING VIDEO MICROSCOPY TO CHARACTERIZE MICROMECHANICS OF BIOLOGICAL AND MAN-MADE MICROMACHINES
}

\author{
Dennis M. Freeman \\ freeman@mit.edu \\ C. Quentin Davis \\ qdavis@mit.edu \\ Department of Electrical Engineering and Computer Science and \\ Research Laboratory of Electronics, \\ Massachusetts Institute of Technology, Cambridge, Massachusetts 02139; \\ Eaton-Peabody Laboratory of Auditory Physiology, \\ Massachusetts Eye and Ear Infirmary, Boston, Massachusetts 02114.
}

\begin{abstract}
Microscopic mechanics at the scale of individual sensory receptor cells play an important signal processing role in the inner ear. However, there are few direct measurements - in large part because the measurements are technically challenging. The important structures are small (micrometers) and the motions are even smaller (nanometers) as well as fast (audio frequencies from $20 \mathrm{~Hz}$ to $20 \mathrm{kHz}$ ). Furthermore, to relate the motions of different structures, simultaneous measurements from many targets are important.

We have developed a new method for visualizing and measuring the sound-induced motions of inner-ear structures. We use (1) a light microscope to magnify the image of the inner ear and to project the image onto a CCD camera, (2) optical sectioning to obtain sequences of images that characterize the entire $3 D$ structure, and (3) stroboscopic illumination to create slow motion video sequences in response to audio-frequency stimuli. Even after optical magnification, sound-induced displacements of inner-ear structures are small compared to the pixel spacing of modem CCD cameras. Nevertheless, subpixel motions modulate the brightness field. We have modified robot vision algorithms to determine motion from changes in the brightness field.

Although this system was designed to study mechanics of the inner ear, it has broader application. We illustrate possible applications in MEMS with measurements of the three-dimensional motions of the teeth in a microfabricated comb drive.
\end{abstract}

\section{BACKGROUND}

During the past 15 years, microelectromechanical systems have evolved from scientific curiosities to viable commercial products, holding promise for revolutionizing the fields of sensor and actuator design. Enormous progress in molecular biology and cell physiology during those same 15 years has enabled scientists to further understand the pervasiveness and elegance of design of biological microelectromechanical systems. Our research group is studying one such system - the cochlea, which is the part of the inner ear that responds to sound.

The cochlea is a remarkable signal processing unit. It has a single mechanical input: the piston-like motion of the stapes, which is the last in the chain of middle ear bones that move when sound strikes the eardrum. Therefore, one could view the cochlea as a simple displacement sensor - albeit, one with extraordinary sensitivity [31; 8]. Using neural signals generated by the cochlea, humans can reliably detect sounds that cause motions of the stapes on the order of picometers - i.e., on the order of the diameter of a hydrogen atom. However, the cochlea is more than a simple displacement sensor [26. Its neural outputs manifest considerable signal processing. The cochlea performs a high-quality frequency analysis of low-level sounds: $Q_{10 d B}>600$ has been recorded for motions in the saline-filled cochlea of the bat [22]. Furthermore, cochlear signal processing is profoundly nonlinear, even at the threshold of hearing. One important nonlinearity is compression. Humans detect sounds differing in intensity over a 120 decibel range. This range of intensities is compressed as it is encoded for neurons with considerably smaller (20-50 dB) dynamic ranges. Our group is interested in understanding the micromechanical mechanisms underlying these signal processing capabilities of the cochlea.

Cochlear mechanics are organized hierarchically. Sensory reception in the cochlea is mediated by hair cells $[16 ; 17]$, which are specialized for mechanoreception by a microscopic bundle of mechanically sensitive hairs (Figure 1). Rotations of a hair bundle cause changes in the intracellular potential of the hair cell, thereby stimulating the release of a neurotransmitter that causes action potentials of cochlear neurons.

The mechanisms by which motions of hair bundles change intracellular potential involve more microscopic mechanical events. Like all cells $[32 ; 33]$, hair cells are surrounded by a cell membrane. The cell membrane consists of a lipid bilayer that acts as an electrical and chemical barrier between the inside and outside of the cell. In ad-

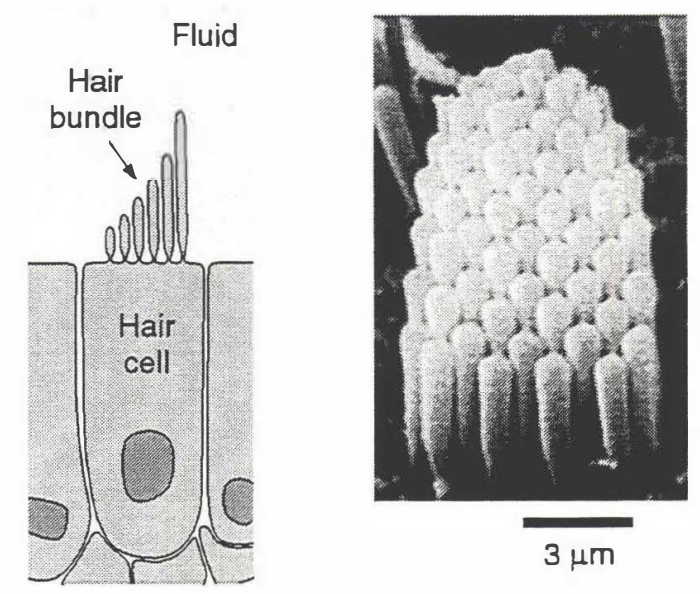

Figure 1. Structure of a hair cell. The left panel shows a schematic diagram of a hair cell and its mechanically sensitive hair bundle. The scanning electron micrograph in the right panel shows a surface view of a hair bundle in the cochlea of the lizard viewed in the direction of the arrow. Each bundle contains 50 to 100 sensory hairs. 


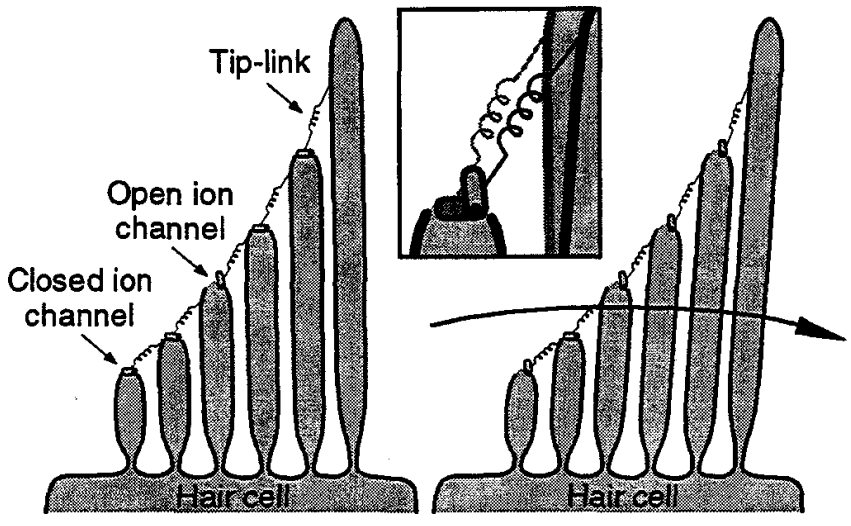

Figure 2. Schematic representation of stress-gated ion channels in hair cells. Rotation of the hair bundle (right) changes the mechanical stress in tip-links, which are represented here as springs connecting adjacent sensory hairs. The change in tip-link stress modulates the ionic conductivity of ion channels, schematized here as trap-doors. The inset shows a magnified view near one tip-link. The gray and black images were taken from the left and right panels, respectively.

dition, the cell membrane contains integral membrane proteins. One class of membrane proteins, the ion channels, act as pores through which ionic currents can pass between the inside and outside of the cell. Hair cells contain ion channels whose state of ionic conductivity is modulated by mechanical stress. The mechanical stress that acts on these stress-gated ion channels is believed to be generated by flaments called tip-links [27; 18], which connect the tips of adjacent sensory hairs (Figure 2).

Fluid dynamics plays a key role in cochlear micromechanics. Hair cells do not operate in isolation but are organized along with other cells and tissues to form a sensory epithelium (Figure 3). Displacements of the stapes cause pressures in the fluids that act to deform the sensory epithelium. As the tissues deform, shearing motions between the hair cells and overlying tectorial membrane cause rotations of hair bundles. Forces of fluid origin play a key role in determining motions of this coupled, hydromechanical system. Interaction of mechanical properties of the epithelium with inertial fluid forces accounts for much of the frequency selectivity exhibited in motion of the epithelium $[25 ; 29 ; 30]$. Fluid forces play a key role in determining the motions of hair bundles $[9 ; 5]$ and contribute to a micromechanical resonance of some hair bundles of lizards [10].

Cochlear structures are extensively cross-coupled. Although organized hierarchically, the levels are not independent. For example, the state (open or closed) of stress-gated ion channels (nanometer scale) has been shown to affect the stiffness of the hair bundle (micrometer scale) [15]. Motions of hair bundles are coupled both mechanically and hydrodynamically to one another and to the sensory epithelium (millimeter scale). Through the surrounding fluids, hair cells can also interact chemically and electrically.

In humans, the cochlear micromachine consists of approximately 15,000 hair cells, each with more than 50 sensory hairs and associated tip-links in a saline-filled environment. The challenge is to understand how these parts - more than a million in each ear - interact to form a system with the remarkable signal processing capabilities of the ear.
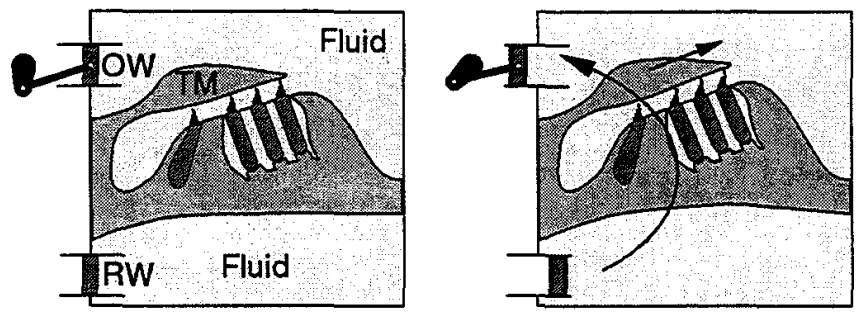

Figure 3. Schematic cross-section of the cochlea. The sensory epithelium and overlying tectorial membrane (TM) separate two fluid-filled compartments. One is coupled to the oval window (OW), into which the piston-like foot of the stapes fits; the other is connected to the round window $(R W)$, which is covered by a thin membrane. The right panel illustrates deformations caused by motions of the stapes.

\section{METHODS}

We have developed a video system to measure sound-induced motions of cochlear structures in an in vitro preparation of the alligator lizard cochlea [6]. The cochlea is mounted in an experimental chamber so that it can be viewed with a microscope while simultaneously being stimulated by an underwater pressure source (Figure 4). The chamber is mounted on the stage of a light microscope (Zeiss Axioplan, New York, NY) that projects magnified images of cochlear structures onto a scientific grade CCD camera (Photometrics 200 series with a Thompson 7883 CCD, Tucson, AZ). The camera has $576 \times 384$ pixels with $23 \mu \mathrm{m}$ spacing. Total magnification between the target and camera can be adjusted from 5 to 600 .

Optical sectioning. Many biological tissues, including hair cells, are nearly transparent. Images can be obtained from any part of such a tissue by altering the microscope's focus control. Thus images from a series of focal depths can be combined into a three-dimensional image that characterizes all of the structures in the cochlea, including hair cells, hair bundles, and the tectorial membrane. Optical sectioning can also be used to generate three-dimensional images of opaque

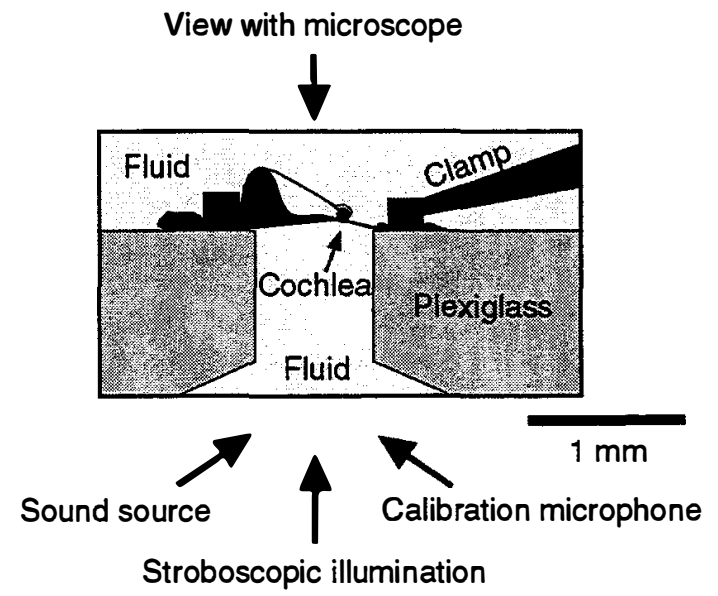

Figure 4. Cross-sectional view of the experimental chamber. The cochlea is clamped so that it seals a circular opening between two fluid-filled compartments. The cochlea is stimulated by a piezoelectric disk that generates hydrodynamic pressures in the bottom compartment; the top compartment is vented to the atmosphere. Sound pressure is calibrated using an underwater microphone located just under the cochlea. 
targets. However, only unoccluded surface structures are visible. To automate the imaging of three-dimensional structure, a computercontrolled stepper motor is used to adjust the focus control of the microscope with a resolution of $1 / 11 \mu \mathrm{m}$ per step.

Stroboscopic illumination. The important motions of cochlear structures are at audio frequencies, i.e., from $20 \mathrm{~Hz}$ to $20 \mathrm{kHz}$. These frequencies exceed the maximum rates of scientific-grade (12-bit) video imagers (it takes 3 seconds for our system to digitize a full frame image and save it to the disk). Therefore, stroboscopic illumination is used to slow the apparent rate of motion. We typically use a gas discharge strobe lamp (Chadwick-Helmuth Co. Inc., El Monte, CA) which generates high intensity illumination. However, the light from a gas discharge strobe is not spatially uniform and pulse-topulse variations can cause motion artifacts. Therefore, light from the strobe is coupled to the microscope through a fiber optic scrambler (Technical Video Ltd., Woods Hole, MA). The scrambler also isolates our system from the considerable electromagnetic interference that is generated by the strobe. The duration of the strobe pulse $(8 \mu \mathrm{s})$ limits the maximum frequency of motion that our system can measure. To relax this constraint, we have recently developed a stroboscopic light source using an LED (AND Division of Purdy Electronics Corp., Sunnyvale, CA). Although they generate less intense light, LEDs radiate much less electromagnetic interference and are orders of magnitude faster.

Subpixel motion estimates from video images. Fundamental limitations on inage resolution and on motion detection from images are different. Image resolution is limited by the optics [12]. To a first approximation, images are blurred by convolution with a point-spread function $[11 ; 2]$, which limits resolution to distances on the order of the wavelength of light (typically $550 \mathrm{~nm}$ ). However, one can detect motions of a blurred image that are significantly smaller. The fundamental limitations on motion detection are determined by the quantum nature of light (shot noise) and by the video imager.

Subpixel displacements can be estimated from changes in brightness (Figure 5) using methods originally developed for robot vision [13]. Generally we identify regions of interest that contain a single moving structure. Images at successive times are then analyzed to determine the best rigid-body translation in a least-squares sense [14].

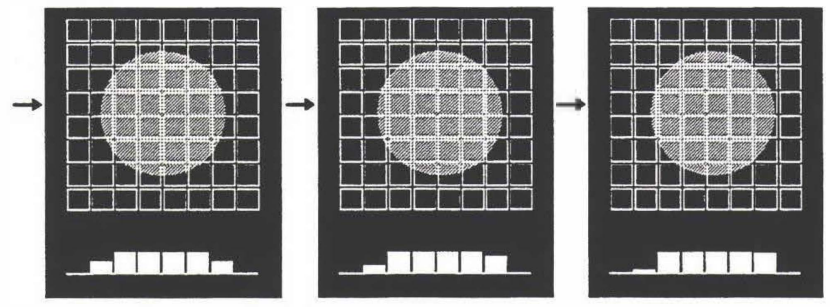

Figure 5. Variations in brightness caused by subpixel motions. The array of squares in each panel represents the pixels of a video camera; the gray disk represents the image of a simple scene; and the bar plots represent the brightness of the row of pixels indicated by the arrow. The three panels illustrate rightward motion of the disk. Although the displacements are subpixel, information about the motion is available from changes in the brightness of the pixels near the edges.

\section{RESULTS}

\section{Sound-Induced Motions of Cochlear Structures}

Figure 6 shows the cochlea of an alligator lizard as imaged with our system. The hair bundles of hair cells and the overlying tectorial membrane are clearly visible. Generally 30 to 60 hair cells are contained in the field of view. Approximately 20 can be seen in Figure 6, which shows only a portion of the camera's field of view.

Because the cochlea is nearly transparent, one can view structures both above and below those shown in Figure 6 by changing the plane of focus. In this experiment, images were acquired at 13 planes of focus separated by $3 \mu \mathrm{m}$. In general, one can obtain sufficiently many images to determine the three-dimensional structure of any visible structure in the cochlea. Figure 7 illustrates how the sequence of two-dimensional images can be combined to obtain a three-dimensional image of a hair bundle and overlying tectorial membrane.

Three-dimensional images of the cochlea were obtained at 8 phases of the sinusoidal stimulus. The resulting data set consisted of 104 images of $576 \times 382$ pixels ( 13 planes times 8 phases) and total data collection time was 5 minutes. This amount of time is large compared to the time needed to determine the motion of a single structure using other measurement methods, such as laser interferometry. However, from the resulting 104 images, one can estimate the threedimensional displacement waveform for every resolvable structure in the field of view. Thus the total measurement time is in fact extraordinarily short compared to the time it would take to make motion measurements of each structure using another method. We illustrate the utility of this feature by showing how two separate analyses of the same data set may be used to address two issues at different spatial scales - the relation between hair bundle motion and that of the overlying tectorial membrane, and relations among the motions of neighboring sensory hairs within a hair bundle.

To determine motions of the hair bundle and overlying tectorial

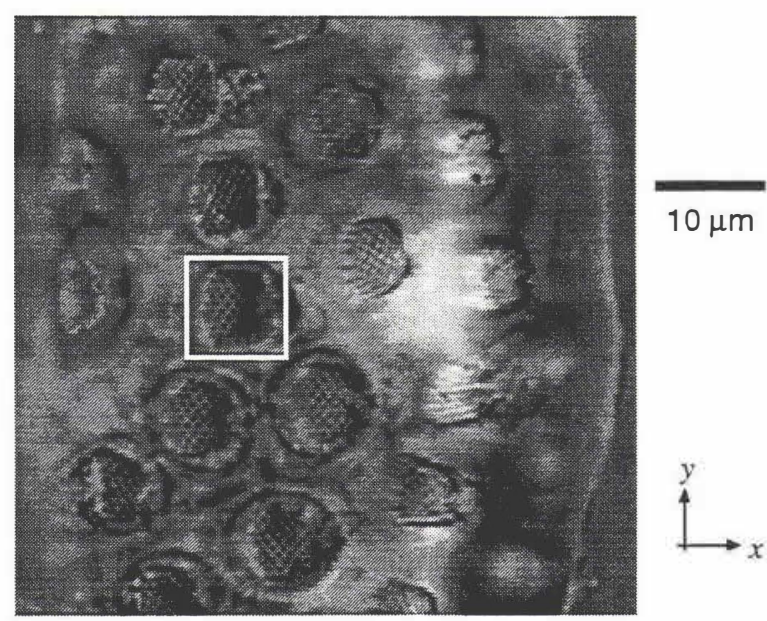

Figure 6. Cochlear structures in the alligator lizard. This figure shows a portion $(240 \times 240$ pixels $)$ of a video image of the lizard cochlea. The white square surrounds one hair bundle; several other hair bundles are also visible. The faint vertical structure at the right edge of the image is the edge of the tectorial membrane (see Figure 3). Total optical magnification from specimen to video camera was $100 \times$. 


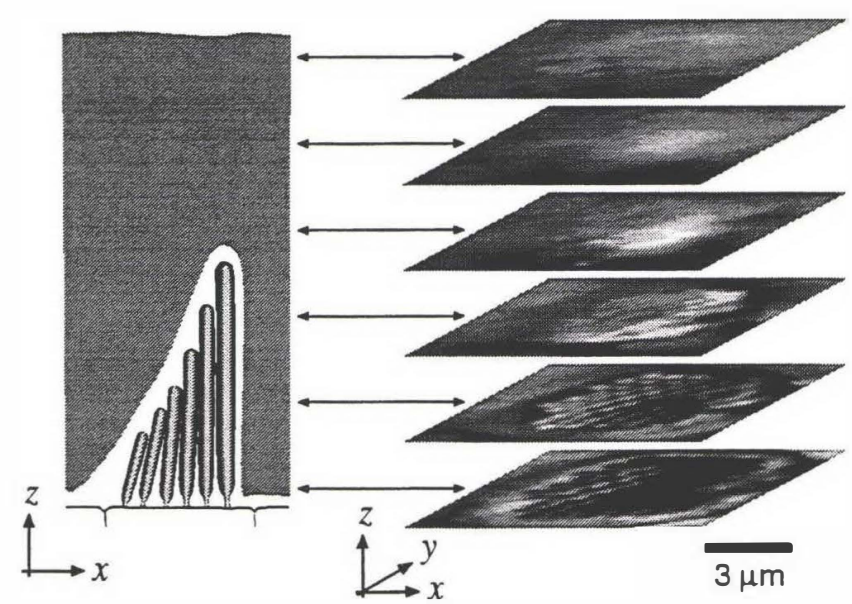

Figure 7. Three-dimensional image of a hair bundle. The bottom $2 D$ image shows a magnified and tilted version of the highlighted part of Figure 6. The other images are from a sequence of planes, each $3 \mu \mathrm{m}$ above the previous one. The double-headed arrows associate each image with the corresponding plane of section in the schematic drawing on the left. The scale bar applies to both the $x$ and $z$ directions; $y$ is foreshortened.

membrane, we analyzed motions of the structures in the 6 images of Figure 7. Displacements near the base of the hair bundle were larger than those near the tip (Figure 8), which demonstrates that the hair bundle is rotating about its point of attachment to the hair cell. Displacements of the tectorial membrane were generally smaller than those of either the base or tip of the hair bundle. Furthermore, as distance from the base of the hair bundle increases, the motion shows increasing phase lag.

To get a quantitative estimate of hair bundle rotation, each displacement waveform was subtracted from the displacement waveform for the base. The results show that the displacement of the tip of the hair bundle relative to the base is $0.3 \mu \mathrm{m}$ peak-to-peak. Since the planes through the tip and base are separated by $6 \mu \mathrm{m}$, hair bundle rotation is approximately $3^{\circ}$ peak-to-peak. Motions of five other hair bundles in this data set were analyzed and the results were qualitatively similar to those shown in Figure 8.

In all of our 12 experiments to date, displacements of the tips and bases of hair bundles have been larger than those of the overlying tectorial membrane for frequencies throughout the lizard's auditory range. This result is consistent with the idea that inertial forces arising from the mass of the tectorial membrane and surrounding fluid tend to resist motion of the tectorial membrane [24]. This result is not consistent with other theories that suggest that the tectorial membrane is a resonant structure [1]. However, our results should be regarded as preliminary. Control experiments must be performed to assure that the cochlea is not damaged during experimentation. Regardless of the ultimate resolution of this particular issue, the important point is that video microscopy can be used to make measurements to directly test theoretical conceptions.

The same data set used in the previous analysis can also be used to study mechanical events at the level of individual sensory hairs. The sinusoidal stimulus generates nearly sinusoidal motions of all the sensory hairs (top right panel of Figure 9). To determine if there is relative motion between the sensory hairs, we applied our motion detection algorithm to estimate the motion of the right edge of the bundle and shifted the images to compensate for that motion. The resulting images show relative motions between sensory hairs (bot-
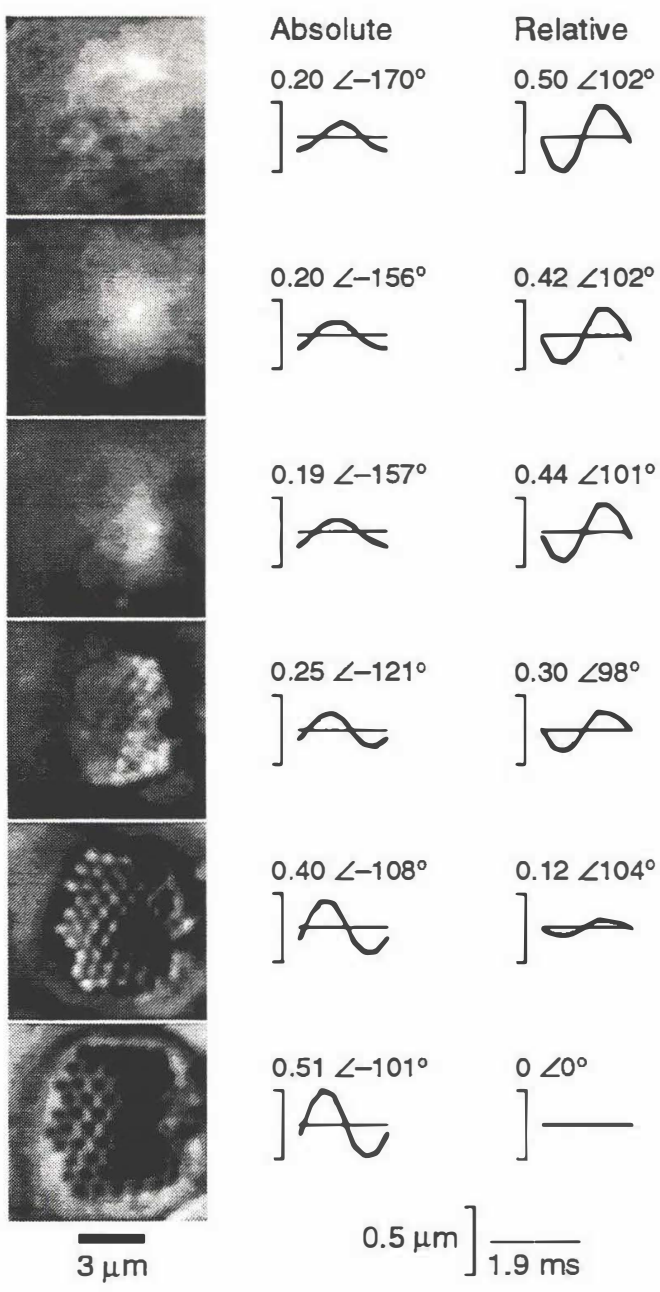

$0.5 \mu \mathrm{m}] \overline{1.9 \mathrm{~ms}}$

Figure 8. Sound-induced motions at six planes of focus through a hair bundle and overlying tectorial membrane. Images (left column) corresponding to those illustrated in Figure 7 were obtained at 8 phases of the stimulus period and were analyzed to quantify motion at each plane of section. The waveforms in the center column show average displacements in the $x$ direction during one cycle of the stimulus. The associated numbers are the peak-to-peak magnitude (in $\mu \mathrm{m}$ ) and angle (in degrees) of the fundamental component of the displacement. The waveforms in the right column show differences between the waveforms in the center column and the bottom waveform in the center column. The intensity of the $513 \mathrm{~Hz}$ stimulus (115 dB SPL in the fluid adjacent to the basilar membrane) corresponds to approximately $89 \mathrm{~dB} S P L$ at the eardrum.

tom right panel of Figure 9).

Although tip-links are too small to be resolved in a light microscope, the results in Figure 9 show that we can directly measure the motions of the sensory hairs to which the tip-links are attached. The relative motions of the tips of the sensory hairs give direct estimates of tip-link stretch, which is the physiologically important mechanical input for transduction mechanisms at the nanometer scale. Although preliminary, our results suggest that tip-links are stretched not only because the distance between insertion points increases when the hairs are rotated by equal angles (as illustrated in Figure 2), but also because the angular displacement of shorter hairs is greater than that of longer hairs. Motions of individual sensory hairs have been 


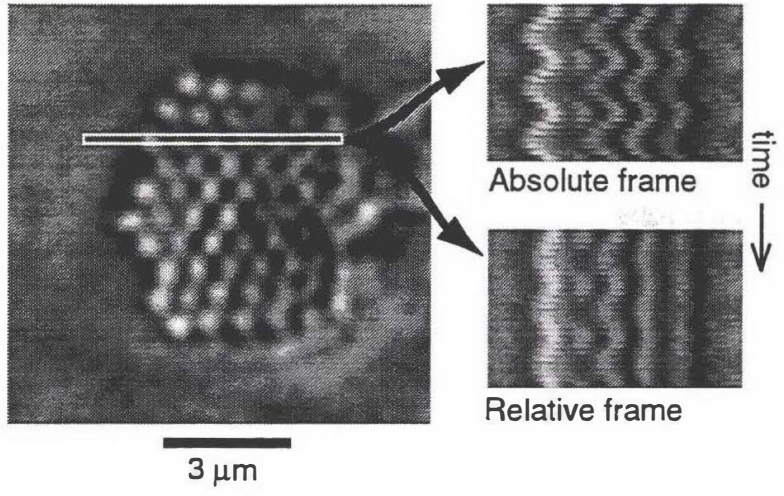

Figure 9. Motions of sensory hairs within a bundle. The left panel is an enlarged image of the hair bundle in Figure 7, at a plane of section near the center of the bundle (second from bottom). The highlighted region was extracted from each of eight images acquired during the stimulus period and positioned so that regions from earlier phases are on top of regions from later phases. The resulting montage, which illustrates motions during one period, is repeated three times to generate the images in the right panels. The upperand lower right panels are similar, except that the images used to generate the lower panel were first shifted to cancel the motion of the right part of the hair bundle.

measured elsewhere $[7 ; 23]$ but only for hair bundles that were stimulated with a water jet; the membranes through which natural stimuli are delivered had been removed.

\section{Three-Dimensional Motions of a Comb Drive}

Although originally developed to measure motions of cochlear structures, our video system has broader application. We have performed several pilot experiments to determine if it can be used to measure the motions of man-made micromechanical devices.

Figure 10 shows an image of a microfabricated accelerometer obtained using our system. The comb drive was driven with an 8.8 $\mathrm{Hz}$ sinusoidal voltage, 44 volts peak-to-peak with an 80 volt DC offset. Images were obtained at 45 planes of focus with $0.45 \mu \mathrm{m}$ spacing to generate a three-dimensional image. Three-dimensional images taken at 8 phases of the sinusoidal stimulus were analyzed to determine the motions of the stationary and fxed teeth highlighted in Figure 10. Displacements of the moving tooth were approximately $2 \mu \mathrm{m}$ peak-to-peak in the horizontal direction (left plot, solid line). These displacements are significantly larger than the corresponding displacements of the fixed tooth, which serve as control measurements to assess the stability of the measurement system. Vertical motions (center plot) were about $6 \mathrm{~nm}$ peak-to-peak for both the fixed and moving teeth, which is near the noise floor of our measurement system (without averaging).

Our system can also measure out-of-plane motions. Although one cannot use optical sectioning to "see through" opaque structures, three-dimensional images of opaque structures still provide information about three-dimensional structure and motion, as illustrated in Figure 11. Image brightness is greatest when the tooth is "in focus" and decreases as the plane of focus is moved higher or lower. Our motion detection algorithm takes advantage of these changes in brighness to align three-dimensional images of the same tooth taken at different phases of the sinusoidal stimulus. Results indicate that the out-of-plane displacement of the moving tooth was $80 \mathrm{~nm}$ peak-

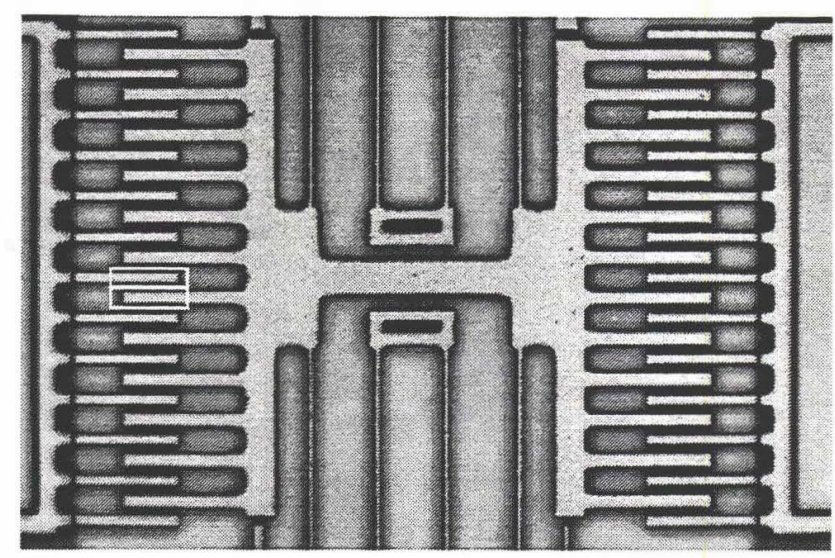

$50 \mu \mathrm{m}$

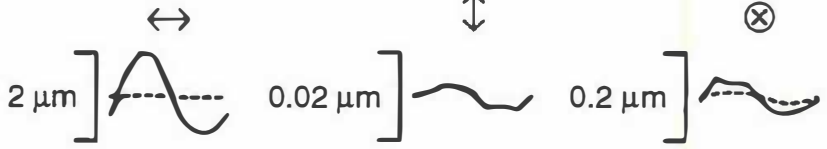

Figure 10. Motions of teeth in a comb drive. The field of view of this image (64x magnification) of a microfabricated accelerometer is focused on the central shuttle and associated comb drives. Most of the folded-beam cantilever that supports the shuttle lies above and below the field of view; only a small portion of it is visible near the center of the image. The portions of the images in the white boxes were analyzed to deternine the motions of a stationary (top) and moving (bottom) tooth of the comb drive, and results are shown in the three plots as dashed and solid curves, respectively. The left, center, and right panels show average displacements in the horizontal, vertical, and out-of-plane directions, respectively.

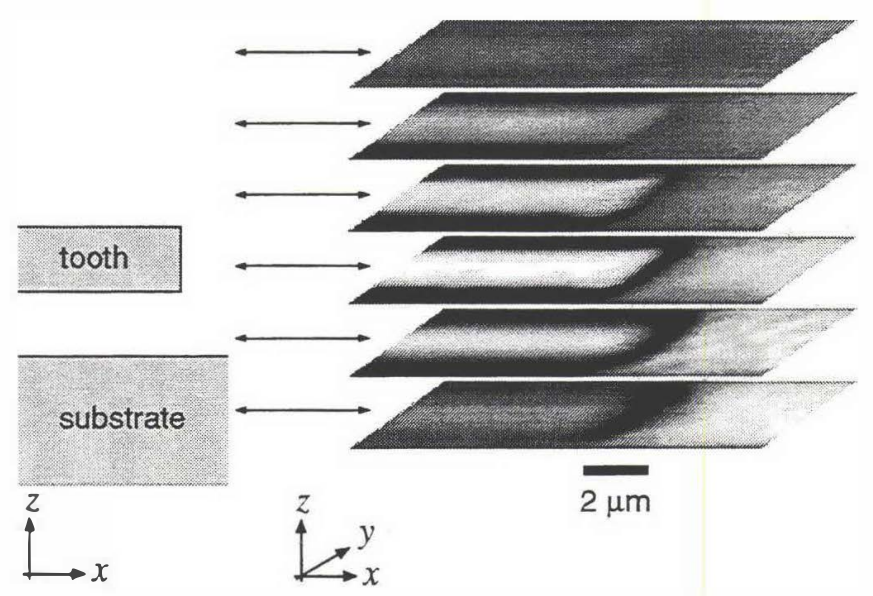

Figure 11. Three-dimensional image of a tooth in a comb drive. This figure illustrates 6 images of the stationary tooth highlighted in Figure 10. The images are at different planes of focus, separated by $2.27 \mu \mathrm{m}$. The double-headed arrows associate each image with the corresponding plane of section in the schematic drawing on the left. The scale bar applies to both the $x$ and $z$ directions; $y$ is foreshortened.

to-peak. The out-of-plane displacement of the stationary tooth was much smaller (26 nm peak-to-peak). 


\section{DISCUSSION}

We have developed a video system that can be used to characterize the motions of both biological and man-made micromechanical systems. Using stroboscopic illumination, we observed motions over the entire audio range of frequencies, and can in principle observe motions at much higher frequencies. Using the optical sectioning property of a light microscope, we measured three-dimensional motions of both transparent (biological) and opaque (silicon) structures. Quantitative results were obtained directly from the video images, based on motion detection algorithms originally developed for robot vision.

\section{Implications for hearing research}

Using the video system, we have obtained the first direct experimental observations of the relation between sound-induced motions of the tectorial membrane and hair bundle displacement. We have also obtained the first direct experimental observations of soundinduced motions of sensory hairs within a bundle. Although these results must be regarded as preliminary until control experiments have tested the viability of our preparations, they demonstrate how video microscopy can be used to probe important cochlear mechanisms at a variety of spatial scales: from ion channels (nanometers) to hair bundles (micrometers) to the entire cochlea (millimeters). Video microscopy has opened the experimental door to understanding key relations in cochlear mechanics that have previously been addressed only theoretically.

\section{Implications for MEMS}

Tools for characterizing the performance of man-made micromachines are primitive by comparison to those available for electronic devices. For many micromachines, tools for assessing internal failure modes do not exist, and only the terminal behaviors can be tested. The inability to characterize internal failure modes has important consequences for both the designer and fabricator. For the designer, it can result in large numbers of prototypes. For the fabricator, it means reduced yields because common techniques such as laser trimming cannot be applied. As the complexity of micromachines increases and as their production becomes a commercial endeavor, developing better tools for characterizing the performance of micromachines will become crucial. Results in this paper demonstrate the potential use of video methods for characterizing the performance of MEMS devices.

\section{Comparison to other motion measurement systems}

A number of systems have been developed to measure micromechanical motions. Our method is different from and has several advantages over previous methods, including the Mössbauer method [19], photodiode method [4], capacitive probe [34], and laser interferometry $[20 ; 28]$. (1) Our motion estimates are derived directly from sequences of images. Thus all of our quantitative results can be checked qualitatively by simply viewing the slow-motion video sequences. For example, to test that the displacement estimates in Figure 8 were qualitatively correct, each image was shifted by the estimated displacement in a direction to compensate for the motion. The resulting sequence of images, which should be stationary, provided a test that motions were estimated properly. (2) In the video method, the identity of the target whose motion is measured is unambiguous since motion is determined directly from images of the target. All the other methods listed above require additional observations to determine which motion is being measured. (3) Unlike any of the other methods listed above, the video method allows simultaneous measurement of motions of many structures in the same specimen. This is a significant advantage when determining interrelations among motions of different structures. (4) The video method provides estimates of all three components of rigid body translation for every structure in the image. The other methods listed above provide an estimate of just one component of motion. (5) In contrast to some methods (e.g., the Mössbauer method, some laser interferometric methods [3] and some photodiode methods [4]) our method does not require any mechanical contact with the moving specimen. Mechanical contact has been shown to affect motions of cochlear structures [21], and it seems inescapable that similar degradations would occur in high-performance man-made micromachines.

The video method has one important disadvantage: it is particularly sensitive to vibrations of the microscope and camera. To reduce vibrations, we use a pneumatic vibration isolation table, which very effectively reduces vibrations at high frequencies but has little effect for low frequencies $(<10 \mathrm{~Hz})$. Although low frequency vibrations contaminate the output signals of all motion detectors, the contamination can generally be filtered out - provided that the frequencies of interest are sufficiently different from the contamination frequencies. However, in the video method, images are acquired at a low rate, typically $0.3 \mathrm{~Hz}$. Low frequency vibrations of the microscope or camera cause displacements of the images that cannot be distinguished from motions near the frequency of interest. These vibrations determine the noise floor of our measurements, which under optimal conditions (e.g., high contrast target, no nearby structures with confounding motions) is approximately $5 \mathrm{~nm}$ without averaging - i.e., $1 \%$ of the wavelength of the light that generates the images. With averaging we have reduced the noise to less than $1 \mathrm{~nm}$. We are currently working on other methods to reduce this noise floor.

\section{ACKNOWLEDGMENTS}

The authors thank Thomas F. Weiss for his contributions throughout this project, RuthAnne Eatock who prepared the scanning electron micrograph shown in Figure 1, and A. J. Aranyosi for helpful comments on the manuscript. This work was supported by grants from the NSF and NIH (R01 DC00238 and P01 DC00119).

\section{REFERENCES}

1. J. B. Allen. Cochlear micromechanics - a physical model of transduction. J. Acoust. Soc. Am., 68:1660-1670, 1980.

2. M. Bom and E. Wolf. Principles of Optics: Electromagnetic Theory of Propagation, Interference and Diffraction of Light. Pergamon Press, New York, 1975.

3. N.P.Cooper and W.S. Rhode. Basilar membrane mechanics in the hook region of the cat and guinea pig cochleae: Sharp tuning and nonlinearity in the absence of baseline position shifts. Hearing Res., 63:163-190, 1992.

4. A. C. Crawford and R. Fettiplace. The mechanical properties of ciliary bundles of turtle cochlear hair cells. J. Physiol., 364:359-379, 1985. 
5. P. Dallos, M. C. Billone, J. D. Durrant, C. Y. Wang, and S. Raynor. Cochlear inner and outer hair cells: Functional differences. Science, 177:356-358, 1972.

6. C. Q. Davis and D. M. Freeman. Direct observations of soundinduced motions of the reticular lamina, tectorial membrane, hair bundles, and individual stereocilia. In Abstracts of the Eighteenth Midwinter Research Meeting, St. Petersburg Beach, Florida, February 1995. Association for Research in Otolaryngology.

7. R. K. Duncan, H. N. Hernandez, and J. C. Saunders. Relative stereocilia motion of chick cochlear hair cells during highfrequency water-jet stimulation. Aud. Neurosci., 1:321-329, 1995.

8. R. R. Fay and A. N. Popper, editors. Comparative Hearing: Mammals in Springer Handbook of Auditory Research. SpringerVerlag, New York, 1994.

9. D. M. Freeman and T. F. Weiss. The role of fluid inertia in mechanical stimulation of hair cells. Hearing Res., 35:201-208, 1988.

10. D. M. Freeman and T. F. Weiss. Hydrodynamic analysis of a two-dimensional model for micromechanical resonance of freestanding hair bundles. Hearing Res., 48:37-68, 1990.

11. J. W. Goodman. Introduction to Fourier Optics. McGrawHill Book Company, New York, 1968.

12. H. H. Hopkins and P. M. Barham. The influence of the condenser on microscopic resolution. Proc. Phys. Soc., 63:737-744, 1950.

13. B. K. P. Horn. Robot Vision. MIT Press, Cambridge, MA, 1986.

14. B. K. P. Horn and E.J. Weldon, Jr. Direct methods for recovering motion. Intematl. J. of Computer Vision, 2:51-76, 1988.

15. J. Howard and A. J. Hudspeth. Compliance of the hair bundle associated with gating of mechanoelectrical transduction channels in the bullfrog's saccular bair cell. Neuron, 1:189-199, 1988.

16. A. J. Hudspeth. The hair cells of the inner ear. Sci. Am., 248:54-64, 1983.

17. A. J. Hudspeth. How the ear's works work. Nature, 341:397404, 1989.

18. A. J. Hudspeth and P. G. Gillespie. Pulling strings to tune transduction: adaptation by hair cells. Neuron, 12:1-9, 1994.

19. B.M. Johnstone and A.J.F. Boyle. Basilar membrane vibration examined with the Mössbauer technique. Science, 158:389390, 1967.
20. S. M. Khanna and D. G. B. Leonard. Basilar membrane tuning in the cat cochlea. Science, 215:305-306, 1982.

21. S. M. Khanna and D. G. B. Leonard. Measurement of basilar membrane vibrations and evaluation of the cochlear condition. Hearing Res., 23:37-53, 1986.

22. M. Kössl and I. J. Russell. Basilar membrane displacement in the cochlea of the mustached bat. In Abstracts of the Eighteenth Midwinter Research Meeting, St. Petersburg Beach, Florida, February 1995. Association for Research in Otolaryngology.

23. R. B. MacDonald and D.P. Corey. Stereocilia bundles of the bullfrog sacculus hair cells do not splay in response to stimulation. In Abstracts of the Nineteenth Midwinter Research Meeting, St. Petersburg Beach, Florida, February 1996. Association for Research in Otolaryngology.

24. F. Mammano and R. Nobili. Biophysics of the cochlea: Linear approximation. J. Acoust. Soc. Am., 93:3320-3332, 1993.

25. S. T. Neely. Finite difference solution of a two-dimensional mathematical model of the cochlea. J. Acoust. Soc. Am., 69(5):1386-1393, 1981.

26. J. O. Pickles. An Introduction to the Physiology of Hearing. Academic Press, San Diego, CA, second edition, 1988.

27. J. O. Pickles and D. P. Corey. Mechanoelectric transduction by hair cells. Trends in Neurosci., 15:254-259, 1992.

28. M. A. Ruggero and N. C. Rich. Application of a commercially-manufactured Doppler-shift laser velocimeter to the measurement of basilar-membrane vibration. Hearing Res., 51:215-230, 1991.

29. W. M. Siebert. Ranke revisited - a simple short-wave cochlear model. J. Acoust. Soc. Am., 56:594-600, 1974.

30. M.A. Viergever and R.J. Diependaal. Quantitative validation of cochlear models using the Liouville-Green approximation. Hearing Res., 21:1-15, 1986.

31. G. von Békésy. Experiments in Hearing. McGraw-Hill, New York, 1960.

32. T. F. Weiss. Cellular Biophysics: Transport. MIT Press, Cambridge, MA, 1996.

33. T. F. Weiss. Cellular Biophysics: Electrical Properties. MIT Press, Cambridge, MA, 1996.

34. J. P. Wilson and J. R. Johnstone. Basilar membrane and middle-ear vibration in guinea pig measured by capacitive probe. J. Acoust. Soc. Am., 57:705-723, 1975. 